\title{
Measurements of Spectroscopic Quadrupole Moments of Neutron Deficient Ir Isotopes and Shape Coexistence in ${ }^{186} \mathrm{Ir}$
}

\author{
G. Seewald, E. Hagn, B. Hinfurtner, and E. Zech \\ Physik Department, Technische Universität München, D-85748 Garching, Germany \\ D. Forkel-Wirth and R. Eder \\ PPE Division, CERN, CH-1211 Geneva 23, Switzerland
}

ISOLDE Collaboration

CERN, CH-1211 Geneva 23, Switzerland

(Received 12 June 1996)

\begin{abstract}
The spectroscopic quadrupole moments of the neutron deficient radioactive Ir isotopes ${ }^{184} \mathrm{Ir},{ }^{186} \mathrm{Ir}^{g}$, ${ }^{186} \mathrm{Ir}^{m},{ }^{187} \mathrm{Ir}$, ${ }^{188} \mathrm{Ir}$, and ${ }^{189} \mathrm{Ir}$ were measured with the technique of quadrupole-interaction-resolved NMR on oriented nuclei. The ratio of quadrupole moments of the $2^{-}$isomeric state ${ }^{186} \mathrm{Ir}^{m}$ and the $5^{+}$ground state ${ }^{186} \mathrm{Ir}^{g}$ points to different nuclear deformations in these states. In addition, we present a new method for the unambiguous measurement of ground state spins. [S0031-9007(96)01746-2]

PACS numbers: 21.10.Ky, 21.60.Ev, 27.70.+q, 76.60.-k
\end{abstract}

The Ir nuclei lie in the transition region between the well-deformed rare earth nuclei and the spherical nuclei near ${ }^{208} \mathrm{~Pb}$. In this transition region, the nuclear deformation may be influenced considerably by the properties of specific single-particle wave functions, and may thus be different for ground states and low-lying isomeric states, even if the energy difference is small.

The ground state configurations of ${ }^{184} \mathrm{Ir}$ and ${ }^{186} \mathrm{Ir}$ have been the subject of many investigations, both theoretically and experimentally [1-8]. From $\beta$-decay studies of ${ }^{186} \mathrm{Ir}$ $\left(I^{\pi}=5^{+}\right)$a low- $K$ ground state configuration had been proposed for ${ }^{186} \operatorname{Ir}[1,2]$, the main components in the wave function given by $I^{\pi} K=5^{+} 1$ and $5^{+} 0$. This can be understood as the coupling of a $\pi 1 / 2^{-}$[541] state (ground state of ${ }^{185} \mathrm{Ir}$ ) and of a $\nu 1 / 2^{-}[510]$ state (ground state of ${ }^{185} \mathrm{Os}$ ), for which $K=0$ and 1 is expected. In the framework of the rotational model the $K$-quantum number can be determined from the spectroscopic quadrupole moment $Q$ which is connected with the intrinsic quadrupole moment $Q_{0}$ by

$$
Q=Q_{0} \frac{3 K^{2}-I(I+1)}{(I+1)(2 I+3)} .
$$

Thus, for a low- $K$ state of a nucleus with prolate deformation, a negative quadrupole moment is expected. For ${ }^{186}$ Ir, this was confirmed by the measurement of the spectroscopic quadrupole moment with quadrupole-interaction nuclear orientation (QI-NO), which yielded $Q=$ $-2.41(20) \mathrm{b}$ [3]. This value indicated a larger nuclear deformation than expected from the extrapolation of heavier Ir isotopes. In this context, it is an interesting question whether the nuclear deformation of the low- $K$ anomalous ground state ${ }^{186} \mathrm{Ir}^{g}$ is enhanced by the specific properties of the $\pi 1 / 2^{-}[541]$ proton intruder state coming down from the $\pi h_{9 / 2}$ orbital. This can be tested by a measurement of the quadrupole moment of the low- lying $I^{\pi} K=2^{-} 2$ isomeric state ${ }^{186} \mathrm{Ir}^{m}$ (configuration: $\left.\pi 3 / 2^{+}[402] \nu 7 / 2^{-}[503]\right)$.

For ${ }^{184} \mathrm{Ir}$, from the $\beta$-decay properties, a similar low- $K$ ground state configuration had been proposed [2]. Here, QI-NO measurements yielded $Q=+2.2(4)$ b [4], which precluded a low- $K$ configuration. It was proposed that the ${ }^{184} \mathrm{Ir}$ ground state is described by a mixture of $K=4$ and 5 components, which can be understood as arising from the coupling of a $\pi 1 / 2^{-}$[541] state (ground state of ${ }^{183} \mathrm{Ir}$ ) and a $\nu 9 / 2^{+}[624]$ state (ground state of ${ }^{183} \mathrm{Os}$ ), for which $K=4$ and 5 is expected. Recently, from spectroscopic investigations, the configuration and even the assignment of $I=5$ for ${ }^{184}$ Ir was doubted [7].

Here, in addition to precision measurements of quadrupole moments, we present a new method for the measurement of ground state spins with resonance precision. It is based on the following features: For the case of a combined magnetic-dipole plus electric-quadrupole hyperfine interaction the resonance spectrum consists of $2 I$ subresonances which are separated equidistantly around the magnetic hyperfine splitting. The frequency offset of the subresonance with the largest amplitude to the magnetic hyperfine splitting depends on $I$. Thus $I$ can be determined by frequency measurements. This method was applied to ${ }^{184} \mathrm{Ir}$, with the unambiguous result $I=5$.

The questions addressed above can be answered by measuring the quadrupole splittings in hcp-Co, from which-without the exact knowledge of the electric field gradient (EFG) of Ir in hcp-Co-highly precise ratios of quadrupole moments are obtained. We also present, however, results of quadrupole-interaction-resolved NMR on oriented nuclei (QI-NMR-ON) measurements on ${ }^{187} \mathrm{Ir}$, ${ }^{188} \mathrm{Ir}$, and ${ }^{189} \mathrm{Ir}$. With the relative quadrupole splittings of $I^{\pi}=3 / 2^{+}{ }^{187} \mathrm{Ir}$ and ${ }^{189} \mathrm{Ir}$ in hcp-Co, and the quadrupole moments of $I^{\pi}=3 / 2^{+}{ }^{191} \mathrm{Ir}$ and ${ }^{193} \mathrm{Ir}$ known from muonic x-ray spectroscopy [9], we are able to determine 
the EFG of Ir in hcp-Co. Thus we can deduce absolute values for the quadrupole moments of ${ }^{184} \mathrm{Ir},{ }^{186} \mathrm{Ir}^{g},{ }^{186} \mathrm{Ir}^{m}$, ${ }^{187} \mathrm{Ir},{ }^{188} \mathrm{Ir}$, and ${ }^{189} \mathrm{Ir}$.

For Ir in a hcp-Co single crystal, in addition to the magnetic hyperfine interaction, there exists an electric quadrupole interaction. The strength of the interactions is specified by the magnetic and electric hyperfine splitting frequencies which are defined as

$$
\begin{gathered}
\nu_{M}=\left|g \mu_{N} B_{\mathrm{HF}} / h\right|, \\
\nu_{Q}=e^{2} q Q / h .
\end{gathered}
$$

Here $g$ and $e Q$ are the nuclear $g$ factor and the spectroscopic quadrupole moment, and $B_{\mathrm{HF}}$ and $e q$ are the magnetic hyperfine field and the EFG, respectively. Both the magnetic hyperfine field and the EFG depend on the angle $\theta$ of the electronic magnetization with respect to the $c$ axis of the Co single crystal. We denote $B_{\mathrm{HF}}(\theta=$ $\left.0^{\circ}\right)=B_{\mathrm{HF}}^{\|}$and $B_{\mathrm{HF}}\left(\theta=90^{\circ}\right)=B_{\mathrm{HF}}^{\perp}$. The case $\theta=$ $0^{\circ}$ - denoted as $0^{\circ}$ geometry - is realized for zero external magnetic field, or if the external magnetic field is applied parallel to the $c$ axis. To realize the case $\theta=90^{\circ}-$ denoted as $90^{\circ}$ geometry - an external magnetic field has to be applied perpendicular to the $c$ axis, $B_{\text {ext }} \geq B_{A}^{\perp}+$ $B_{\text {dem }}$ where $B_{A}^{\perp}=13.4 \mathrm{kG}$ is the perpendicular anisotropy field and $B_{\mathrm{dem}}$ is the demagnetization field which depends on the dimensions of the sample. (Typical values for $B_{\mathrm{dem}}$ are $0.3 \ldots 0.5 \mathrm{kG}$ in the present work.)

The hyperfine interaction of radioactive Ir isotopes in hcp-Co can be determined with the technique of NMR on oriented nuclei [10]. For an isotope with spin $I$, there exists a set of $2 I$ subresonances. For $0^{\circ}$ geometry (denoted by $\|$ ) and $90^{\circ}$ geometry (denoted by $\perp$ ), the center of the subresonance corresponding to rf transitions between state $|m\rangle$ and $|m+1\rangle$ is, assuming that the $m=I$ state lies lowest in energy, given by

$$
\begin{aligned}
\nu_{m \rightarrow m+1}^{\|}= & \nu_{\mathrm{mag}}^{\|}-\Delta \nu_{Q}^{(\|)}\left(m+\frac{1}{2}\right), \\
\nu_{m \rightarrow m+1}^{\perp}= & \nu_{\mathrm{mag}}^{\perp}-\Delta \nu_{Q}^{(\perp)}\left(m+\frac{1}{2}\right)\left(1+C_{m}^{m+1}\right), \\
\nu_{\mathrm{mag}}^{(\|, \perp)}= & \nu_{M}^{(\|, \perp)}+\left[|g| \mu_{N} \operatorname{sgn}\left(B_{\mathrm{HF}}^{(\|,, \perp)}\right) / h\right] \\
& \times\left(B_{\mathrm{ext}}-B_{\mathrm{dem}}\right)(1+K), \\
\Delta \nu_{Q}^{(\|)}= & \Delta \nu_{Q}^{(c)}+\Delta \nu_{Q}^{(L)} \\
\Delta \nu_{Q}^{(\perp)}= & \Delta \nu_{Q}^{(c)}-\frac{1}{2} \Delta \nu_{Q}^{(L)} \\
\Delta \nu_{Q}^{(c, L)}= & 3 \nu_{Q}^{(c, L)} /[2 I(2 I-1)], \\
\nu_{Q}^{(c, L)}= & e^{2} q^{(c, L)} Q / h .
\end{aligned}
$$

Here $e q^{(c)}$ is the "collinear" EFG which is axial symmetric with respect to the direction of $\vec{B}_{\mathrm{HF}}$ and $e q^{(L)}$ is the lattice EFG which is axial symmetric with respect to the $c$ axis. For $90^{\circ}$ geometry, if the quadrupole interaction due to the lattice EFG is small with respect to the magnetic interaction, $\nu_{Q}^{(L)} \ll \nu_{M}$, the quadrupole subresonance spectrum is still equidistant, and the quadrupole sub- resonance separation is $\Delta \nu_{Q}^{(c)}-\frac{1}{2} \Delta \nu_{Q}^{(L)}$. If $\left|\nu_{Q}^{(L)}\right|$ is not small compared to $\nu_{M}$, the quadrupole subresonance spectrum is no more equidistant. This is taken into account by the correction factors $C_{m}^{m+1}$ in Eq. (5) which are calculated by diagonalization of the hyperfine Hamiltonian. For the present case, the $C_{m}^{m+1}$ are of the order of $10^{-2} \cdots 10^{-1}$. $K$ in Eq. (6) is the resonance shift parameter which takes into account the Knight shift and diamagnetic shielding.

We denote the subresonance between the energetically lowest sublevels as $\nu_{1}$ resonance, the next $\nu_{2}$, etc. At low temperatures, the $\nu_{1}$ resonance has the largest amplitude and can thus be measured with the best accuracy. If higher subresonances can also be observed with sufficient statistical accuracy, the quadrupole interaction is obtained directly from the frequency differences. The highest accuracy is normally achieved for $0^{\circ}$ geometry, but the resonance amplitudes are much larger in $90^{\circ}$ geometry because of the resonancelike enhancement factor for the radio frequency field [11]. If higher subresonances cannot be observed - as for ${ }^{186} \mathrm{Ir}^{m}$ in the present casethe quadrupole interaction can be determined from the measurement of $\nu_{1}$ in $0^{\circ}$ and $90^{\circ}$ geometry,

$$
\nu_{1}^{\|}-\nu_{1}^{\perp}=\Delta \nu_{\mathrm{mag}}-\left(\frac{3}{2} \Delta \nu_{Q}^{(L)}-C_{I-1}^{I} \Delta \nu_{Q}^{(\perp)}\right)\left(I-\frac{1}{2}\right),
$$

where the (small) magnetic contribution to the offset $\Delta \nu_{\text {mag }}$ is obtained via Eq. (6).

In addition, the spin of the oriented state can be determined with resonance measurements: The offset of $\nu_{1}$ to the magnetic hyperfine splitting is given by

$$
\nu_{M}-\nu_{1}=(I-1 / 2) \Delta \nu_{Q} .
$$

Thus the spin of the oriented state can be determined from three frequency measurements,

$$
I=\left(\nu_{\operatorname{mag}}-\nu_{1}\right) / \Delta \nu_{Q}+1 / 2,
$$

where the magnetic splitting $\nu_{\text {mag }}$ can be determined with several kinds of experiments, e.g., if $B_{\mathrm{HF}}^{(\mathrm{Co})} / B_{\mathrm{HF}}^{(\mathrm{Fe}, \mathrm{Ni})}$ is known, by additional NMR-ON measurements with Fe or $\mathrm{Ni}$ as host lattice.

The IrCo samples were prepared at the mass separator ISOLDE at CERN. Hg precursor isotopes were implanted with $E=60 \mathrm{keV}$ into disk-shaped hcp-Co single crystals (diameter $\sim 10 \mathrm{~mm}$; thickness $\sim 0.2 \mathrm{~mm}$ ). Much attention was paid to a high surface quality which was obtained by a sophisticated procedure of mechanical, chemical, and electropolishing steps [12]. The $c$ axis was oriented parallel to the plane of the disks. Using a ${ }^{3} \mathrm{He}-{ }^{4} \mathrm{He}$ dilution refrigerator with a top-loading facility, the samples were cooled to temperatures near $10 \mathrm{mK}$. The radio frequency was frequency modulated, with a band width between \pm 0.05 and $\pm 1.0 \mathrm{MHz}$.

QI-NMR-On spectra of ${ }^{186} \mathrm{Ir}^{g} \mathrm{Co}$ are shown in Fig. 1, for $0^{\circ}$ geometry (top) and $90^{\circ}$ geometry (bottom). In both 


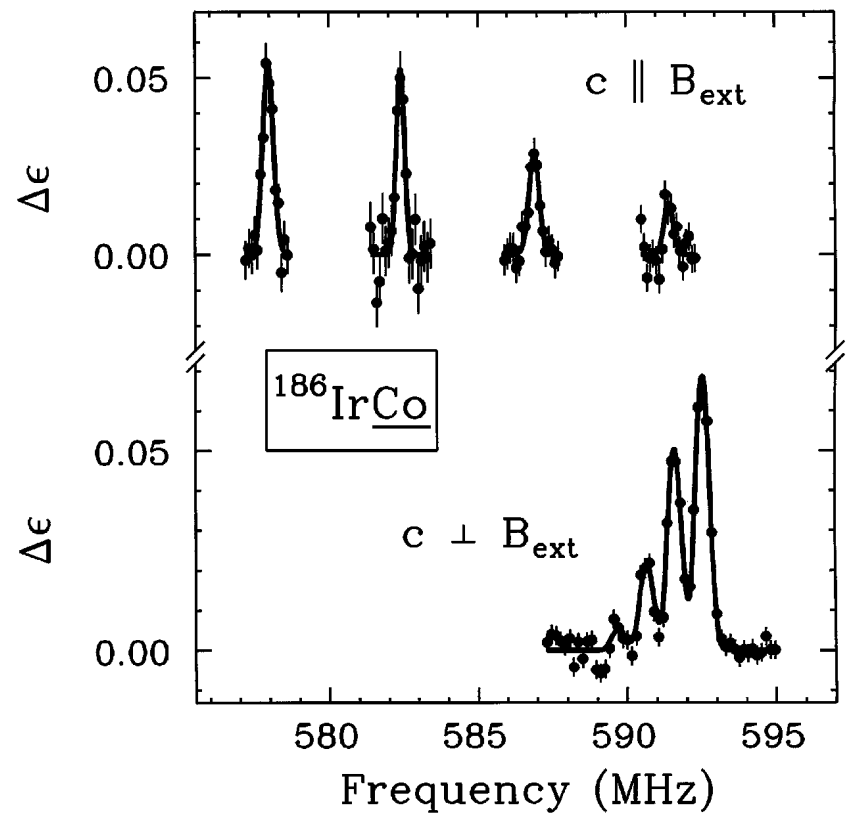

FIG. 1. QI-NMR-ON resonance of ${ }^{186} \mathrm{Ir}^{g} \mathrm{Co}^{\text {(hcp) }}$ in $0^{\circ}$ (top) and $90^{\circ}$ geometry (bottom).

cases, the lowest 4 subresonances are well resolved. The ratio of the $0^{\circ}$ to $90^{\circ}$ subresonance separation $\Delta \nu_{Q}^{\|} / \Delta \nu_{Q}^{\perp}$ differs strongly from -2 , which would be expected for a pure lattice EFG. It indicates the existence of a relatively large collinear EFG. Consistent data with similarly high precision were also obtained for ${ }^{184} \mathrm{Ir},{ }^{187} \mathrm{Ir}$, ${ }^{188} \mathrm{Ir}$, and ${ }^{189} \mathrm{Ir}$. The results are compiled in Table I. For ${ }^{186} \mathrm{Ir}^{m}$ only the $\nu_{1}$ resonance could be observed, however, both for $0^{\circ}$ geometry and $90^{\circ}$ geometry. Taking into account $\Delta \nu_{Q}^{\|} / \Delta \nu_{Q}^{\perp}$ as known from the other isotopes, the respective quadrupole splitting could be determined also with high precision. The $0^{\circ}$ geometry quadrupole splittings and ratios of quadrupole moments are listed in columns 3 and 4 of Table II.

For the derivation of the absolute values of the quadrupole moments the EFG of $\mathrm{IrCo}$ has to be known. Here actually a problem arises as there is no unique link to ${ }^{191} \mathrm{Ir}$ or ${ }^{193} \mathrm{Ir}$ for which the quadrupole moments are known from muonic x-ray spectroscopy [9]. The link via the quadrupole splitting of a radioactive isotope in Fe or $\mathrm{Ni}$ (measured with NMR-ON) to the quadrupole splitting of ${ }^{191} \mathrm{Ir}$ and ${ }^{193} \mathrm{Ir}$ in $\mathrm{Fe}$ and $\mathrm{Ni}$ (measured with conventional NMR) may contain an unpredictable uncertainty, as has been shown recently for $\mathrm{Au}$ in Fe [13]. Thus we apply the procedure with which the quadrupole splittings of $\mathrm{Au}$ isotopes were interpreted correctly [14], as proven later by subsequent laser-spectroscopy measurements [15]. The quadrupole moments of $3 / 2^{+} \mathrm{Au}$ isotopes ${ }^{191} \mathrm{Au},{ }^{193} \mathrm{Au},{ }^{195} \mathrm{Au}$, and ${ }^{197} \mathrm{Au}$ show a nearly perfect linear dependence on $A$ (see Fig. 2). The $3 / 2^{+}$isotopes ${ }^{187} \mathrm{Ir},{ }^{189} \mathrm{Ir},{ }^{191} \mathrm{Ir}$, and ${ }^{193} \mathrm{Ir}$ have essentially the same nuclear structure. This is also supported by the known nuclear magnetic moments which differ only weakly. The measured ratio $Q\left({ }^{187} \mathrm{Ir}\right) / Q\left({ }^{189} \mathrm{Ir}\right)=1.072(2)$ is in excellent agreement with the linear extrapolation from ${ }^{191} \mathrm{Ir}$ and ${ }^{193} \mathrm{Ir}$, according to which 1.074(10) is expected. In this way $Q\left({ }^{189} \mathrm{Ir}\right)=+0.878(10) \mathrm{b}$ is obtained which is used to deduce the quadrupole moments of the other Ir isotopes. These are listed in column 5 of Table II. The intrinsic quadrupole moments deduced via Eq. (1) are shown in Fig. 2. For the even isotopes with "normal" ground state configuration $K=I-{ }^{186} \mathrm{Ir}^{m}$, and ${ }^{188} \mathrm{Ir}$, and ${ }^{192} \mathrm{Ir}-Q_{0}$ is slightly larger than $Q_{0}$ of the odd $I^{\pi}=3 / 2^{+}$isotopes, with the same decreasing trend as a function of $A$.

For ${ }^{184} \mathrm{Ir}$ and ${ }^{186} \mathrm{Ir}^{g}, Q_{0}$ cannot be obtained directly because of the unknown $K$-quantum number. For ${ }^{184} \mathrm{Ir}$, we obtain $Q_{0}=+4.17(5) \mathrm{b}$ for $K=5$ and $Q_{0}=$ $+10.4(1)$ b for $K=4$, with the actual value due to $K$ mixing in between. Thus a detailed knowledge of the ground state wave function would be necessary to deduce $Q_{0}$. For the determination of the ground state spin of ${ }^{184}$ Ir the magnetic hyperfine splitting in $\mathrm{Fe}$ was measured to be 143.6(2) MHz. With $B_{\mathrm{HF}}^{(\mathrm{Co}, \|)} / B_{\mathrm{HF}}^{(\mathrm{Fe})}=0.7538(2), \nu_{1}^{\|}$ 127.171(45) $\mathrm{MHz}$, and $\Delta \nu_{Q}^{\|}=-4.249(32) \mathrm{MHz}$ (see Table I), we get, according to Eq. (13), $I=4.95(6)$. This proves unambiguously $I=5$.

Concerning the deformation, more stringent conclusions can be drawn for ${ }^{186} \mathrm{Ir}^{g}$. We obtain $Q_{0}=+6.62(8) \mathrm{b}$ for $K=0$ and $Q_{0}=+7.36(9)$ b for $K=1$, the actual value due to $K$ mixing again in between, however, with a lower limit, $Q_{0}\left({ }^{186} \mathrm{Ir}^{g}\right) \geq+6.62(8) \mathrm{b}$. This is considerably larger than $Q_{0}$ of the isomeric state, for which, taking $K=I=2, Q_{0}\left({ }^{186} \operatorname{Ir}^{m}\right)=+5.10(6) \mathrm{b}$ is obtained.

TABLE I. Measured resonance frequencies of Ir isotopes in hcp-Co.

\begin{tabular}{llllrrr}
\hline \hline Isotope & $I^{\pi}$ & $\nu_{1}^{\|}(\mathrm{MHz})$ & $\Delta \nu_{Q}^{\|}(\mathrm{MHz})$ & $\nu_{1}^{\perp}(\mathrm{MHz})$ & $\Delta \nu_{Q}^{\perp}(\mathrm{MHz})$ & $\Delta \nu_{Q}^{\|} / \Delta \nu_{Q}^{\perp}$ \\
\hline${ }^{184} \mathrm{Ir}$ & $5^{(-)}$ & $127.171(45)^{a}$ & $-4.249(32)^{a}$ & $101.856(26)^{c}$ & $+0.968(16)^{c}$ & $-4.39(8)$ \\
${ }^{186} \mathrm{Ir}^{g}$ & $5^{+}$ & $577.950(10)^{a}$ & $+4.470(17)^{a}$ & $592.540(10)^{c}$ & $-0.974(11)^{c}$ & $-4.59(6)$ \\
${ }^{186} \mathrm{Ir}^{m}$ & $2^{-}$ & $277.654(16)^{a}$ & & $238.123(62)^{c}$ & \\
${ }^{187} \mathrm{Ir}$ & $3 / 2^{+}$ & $117.876(8)^{a}$ & $-24.719(28)^{a}$ & $85.950(90)^{d}$ & \\
${ }^{188} \mathrm{Ir}$ & $1^{-}$ & $257.229(23)^{a}$ & $-38.102(35)^{b}$ & $229.854(32)^{c}$ & $+8.750(80)^{c}$ & $-4.35(5)$ \\
${ }^{189} \mathrm{Ir}$ & $3 / 2^{+}$ & $100.136(16)^{a}$ & $-23.064(49)^{a}$ & $70.444(90)^{d}$ & $+5.353(79)^{d}$ & $-4.31(6)$ \\
\hline \hline
\end{tabular}

${ }^{a} B_{\text {ext }}=0 \mathrm{kG} ;{ }^{b} B_{\text {ext }}=10 \mathrm{kG} ;{ }^{c} B_{\text {ext }}=18 \mathrm{kG} ;{ }^{d} B_{\text {ext }}=20 \mathrm{kG}$. 
TABLE II. Quadrupole moments of Ir isotopes. (The final values for $\nu_{Q}^{\|}$were obtained from a simultaneous least-squares fit taking into account $\nu_{Q}^{\|}, \nu_{Q}^{\perp}, \nu_{1}^{\|}$, and $\nu_{1}^{\perp}$ of all isotopes.)

\begin{tabular}{clccc}
\hline \hline Isotope & $I^{\pi}$ & $\nu_{Q}^{\|}(\mathrm{MHz})$ & $Q / Q\left({ }^{189} \mathrm{Ir}\right)$ & $Q(\mathrm{~b})$ \\
\hline${ }^{184} \mathrm{Ir}$ & $5^{(-)}$ & $-126.31(43)$ & $+2.742(11)$ & $+2.407(29)$ \\
${ }^{186} \mathrm{Ir}^{g}$ & $5^{+}$ & $+133.69(43)$ & $-2.902(11)$ & $-2.548(31)$ \\
${ }^{186} \mathrm{Ir}^{m}$ & $2^{-}$ & $-76.41(16)$ & $+1.658(5)$ & $+1.456(17)$ \\
${ }^{187} \mathrm{Ir}$ & $3 / 2^{+}$ & $-49.40(5)$ & $+1.072(2)$ & $+0.941(11)$ \\
${ }^{188} \mathrm{Ir}$ & $1^{-}$ & $-25.41(2)$ & $+0.5515(11)$ & $+0.484(6)$ \\
${ }^{189} \mathrm{Ir}$ & $3 / 2^{+}$ & $-46.07(8)$ & 1.0 & $+0.878(10)$ \\
${ }^{191} \mathrm{Ir}$ & $3 / 2^{+}$ & $\ldots$ & $\ldots$ & $+0.816(9)$ \\
${ }^{192} \mathrm{Ir}$ & $4^{(-)}$ & $\ldots$ & $+2.445(58)$ & $+2.147(56)$ \\
${ }^{193} \mathrm{Ir}$ & $3 / 2^{+}$ & $\ldots$ & $\ldots$ & $+0.751(9)$ \\
\hline \hline
\end{tabular}

The deformation parameter $\beta_{2}$ can be calculated using the rotational-model relationship

$$
\beta_{2}=\frac{\sqrt{5 \pi}}{3 Z R_{0}^{2}} \frac{(I+1)(2 I+3)}{3 K^{2}-I(I+1)} Q,
$$

with the results $\beta_{2}\left({ }^{186} \mathrm{Ir}^{m}\right)=+0.17$ and $\beta_{2}\left({ }^{186} \mathrm{Ir}^{g}\right) \geq$ $+0.22 \ldots+0.25$. Thus - despite the very small energy difference between isomer and ground state of $<1.5 \mathrm{keV}$ [8] - the deformation of ${ }^{186} \mathrm{Ir}^{g}$ is considerably larger than the deformation of ${ }^{186} \mathrm{Ir}^{m}$. This is probably connected with the specific properties of the $\pi 1 / 2^{-}$[541] proton intruder state coming down from the $\pi h_{9 / 2}$ orbital: Because of the large negative energy dependence of the $\pi 1 / 2^{-}$[541] single-particle energy with deformation, a larger ground state deformation is favored. Concerning the low-energy level scheme it has been reported that the best agreement between theoretical description and

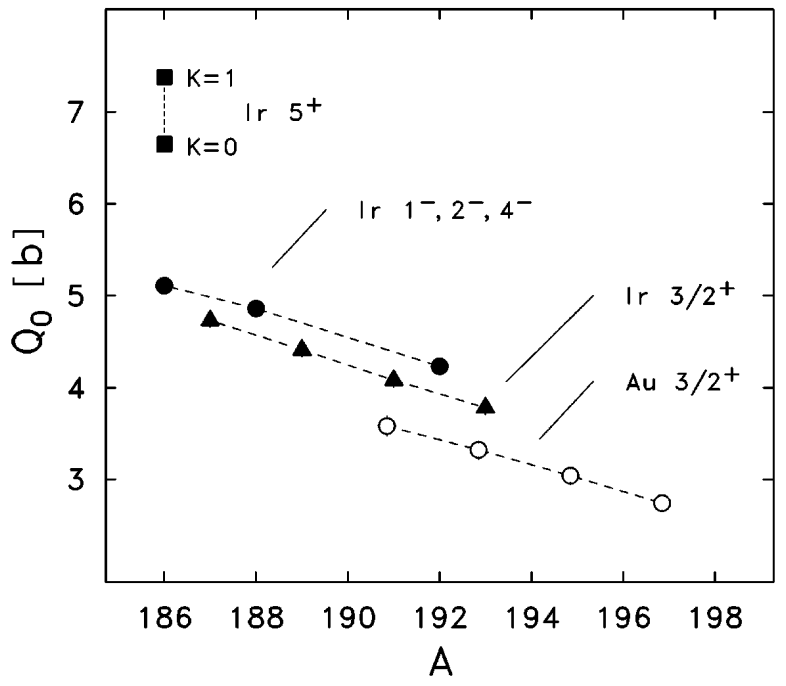

FIG. 2. Intrinsic quadrupole moments of Ir and $\mathrm{Au}$ isotopes. The quadrupole moment of ${ }^{192} \mathrm{Ir}$ is taken from Ref. [16]. experiment has been obtained for $\beta_{2}=0.15$ [8]. The large ground state deformation as observed here is, however, not described properly. Thus, the new data on the quadrupole moments should stimulate a new theoretical approach. In this context it should be added that the correct description of ${ }^{186} \mathrm{Ir}^{g}$ would be a challenge as it is to our knowledge the only one known isotope with a $I-K \geq 4$ anomalous ground state.

We appreciate very much the effort which was put by the ISOLDE and Orsay group into the development of the liquid $\mathrm{Pb}$ target. We also wish to thank E. Smolic for experimental help. This work has been funded by the Deutsche Forschungsgemeinschaft (DFG) under Contract No. Ha 1282/3-3.

[1] K. J. Hofstetter, T. T. Sugihara, and D. S. Brenner, Phys. Rev. C 8, 2442 (1973).

[2] G. T. Emery, R. Hochel, P. J. Daly, and K. J. Hofstetter, Nucl. Phys. A211, 189 (1973).

[3] H. Ernst, E. Hagn, U. Schneider, and E. Zech, Phys. Lett. 86B, 154 (1979).

[4] E. Hagn, H. Kleebauer, and E. Zech, Phys. Lett. 104B, 365 (1981).

[5] A. Ben Braham, C. Bourgeois, P. Kilcher, B. Roussière, J. Sauvage, M. G. Porquet, A. J. Kreiner, and ISOCELE Collaboration, Nucl. Phys. A482, 553 (1988).

[6] A. J. Kreiner, J. Davidson, and M. Davidson, P. Thierberger, E. K. Warburton, S. André, and J. Genevey, Nucl. Phys. A489, 525 (1988).

[7] M. G. Porquet, C. Bourgeois, P. Kilcher, B. Roussière, J. Sauvage, H. Dautet, J. K. P. Lee, and ISOCELE Collaboration, Nucl. Phys. A499, 495 (1989).

[8] A. Ben Braham, C. Bourgeois, P. Kilcher, F. Le Blanc, B. Roussière, J. Sauvage, A. J. Kreiner, M. G. Porquet, and ISOCELE Collaboration, Nucl. Phys. A533, 113 (1991).

[9] Y. Tanaka, R. M. Steffen, E. B. Shera, W. Reuter, M. V. Hoehn, and J. D. Zumbro, Phys. Rev. Lett. 51, 1633 (1983); Phys. Rev. C 29, 1830 (1984).

[10] E. Matthias and R. J. Holliday, Phys. Rev. Lett. 17, 897 (1966).

[11] B. Hinfurtner, E. Hagn, E. Zech, R. Eder, and ISOLDE Collaboration, Phys. Rev. Lett. 64, 2188 (1990).

[12] H. Ratai, diploma thesis, TU Munich, 1991 (unpublished).

[13] B. Hinfurtner, C. König, E. Hagn, E. Zech, R. Eder, D. Forkel, and ISOLDE Collaboration, Nucl. Phys. A562, 205 (1993).

[14] B. Hinfurtner, E. Hagn, E. Zech, R. Eder, NICOLE Collaboration, and ISOLDE Collaboration, Phys. Rev. Lett. 67, 812 (1991).

[15] G. Passler, J. Rikovska, E. Arnold, H.-J. Kluge, L. Monz, R. Neugart, H. Ravn, K. Wendt, and ISOLDE Collaboration, Nucl. Phys. A580, 173 (1994).

[16] G. Seewald et al. (to be published). 